\title{
Proteoglycans and hyaluronan in human fetal membranes
}

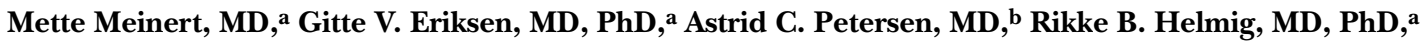 \\ Claude Laurent, $\mathrm{MD}, \mathbf{P h D},{ }^{\mathrm{c}}$ Niels Uldbjerg, $\mathrm{MD}, \mathbf{P h D},{ }^{\mathrm{a}}$ and Anders Malmström, $\mathbf{P h D}^{\mathrm{d}}$ \\ Aarhus and Aalborg, Denmark, and Umeå and Lund, Sweden
}

\begin{abstract}
OBJECTIVE: The aim of this study was to describe the distributions of major extracellular matrix components, such as proteoglycans, collagen and hyaluronan, in the fetal membranes at term.

STUDY DESIGN: Fetal membranes were obtained from elective cesarean deliveries at term. Guanidinium extracts were analyzed for proteoglycans with alcian blue precipitation, sodium dodecyl sulfatepolyacrylamide gel electrophoresis, and Western blotting and for hyaluronan with a radioimmunoassay. Collagen was measured by estimating hydroxyproline content. Tissue sections were immunostained for decorin and biglycan and stained for hyaluronan with a biotin-labeled hyaluronan-binding protein. RESULTS: The fetal membranes contained predominantly smaller proteoglycans, such as biglycan and decorin. The amnion consisted of typical fibrous connective tissue with a high concentration of collagen. The amnion was dominated by decorin located in close connection with the collagen fibrils. The chorion was composed of a fibroblastic part containing collagen and decorin and a trophoblastic part mainly containing biglycan. In addition, large amounts of hyaluronan were found, especially in the amnion and in the decidual cell layers.

CONCLUSION: The distributions of proteoglycans, collagen, and hyaluronan in human fetal membranes may explain the biomechanical properties of this tissue. We suggest that changes in the relative proportions of these extracellular molecules are crucial for the proposed maturation process in the fetal membranes during the last weeks of pregnancy. (Am J Obstet Gynecol 2001;184:679-85.)
\end{abstract}

Key words: Amnion, biglycan, chorion, collagen, decorin, premature rupture of membranes

During pregnancy the fetal membranes play an important role, functioning as a barrier and thereby protecting the fetus in utero. If the fetal membranes fail to maintain their mechanical integrity, preterm prelabor rupture of membranes, a condition associated with high risks of maternal morbidity and neonatal morbidity and mortality, occurs. ${ }^{1}$ The fetal membranes consist of 2 layers, the amnion and the chorion. Biochemical and biomechanical studies have shown that the amnion is the strength-bearing component, ${ }^{2}$ with high contents of collagens type I and III. ${ }^{3}$ Investigations of the fetal membranes during the last weeks of pregnancy have shown a reduced strength of the fetal membranes after vaginal delivery at 40 weeks' gesta-

From the Research Laboratory, Department of Obstetrics and Gynecology, Aarhus University Hospital, a the Department of Pathology, Aalborg University Hospital, ${ }^{b}$ the Department of Clinical Science, University of Umeå, ${ }^{c}$ and the Department of Cell and Molecular Biology, University of Lund. ${ }^{d}$

Supported by grants from The Danish Medical Research Council, The Institute of Clinical Experimental Research, the University of Aarhus, NorFa, The Danish Medical Association Fond, The Swedish Medical Research Council, Astra-Draco AB, and the Codan Insurance Research Foundation.

Received for publication February 10, 2000; revised June 27, 2000; accepted July 21, 2000.

Reprint requests: Mette Meinert, $M D$, Research Laboratory, Department of Obstetrics and Gynecology, Skejby Hospital, 8200 Aarhus N, Denmark.

Copyright @ 2001 by Mosby, Inc.

$0002-9378 / 2001 \$ 35.00+0 \quad 6 / 1 / 110294$

doi:10.1067/mob.2001.110294 tion relative to membranes from cesarean deliveries at 38 weeks' gestation. ${ }^{2}$ This decrease in strength was not associated with a simultaneous decrease in the concentration of collagen. ${ }^{4}$ The role of collagen in preterm prelabor rupture of membranes remains uncertain, because the findings have been contradictory. 5,6

These findings indicate that other extracellular matrix components may be of importance in the final weakening process of the fetal membranes toward term or during active labor. In addition to collagen, another structural component that might be of importance in generating tensile strength is the small proteoglycan decorin, which binds to collagen types I and III and thereby enables lateral organization of the collagen fibrils. ${ }^{7}$ The fact that the tensile strength of the skin of decorin-deficient mice is decreased supports this hypothesis. ${ }^{8}$ Biglycan, another small proteoglycan, is preferentially localized in the pericellular matrix, where it interacts with type VI collagen. ${ }^{9}$ Both decorin and biglycan bind and abrogate the activity of transforming growth factor $\beta .{ }^{10}$ Proteoglycans are composed of a protein core to which one or more sulfated glycosaminoglycan side chains are attached. ${ }^{10}$ In addition to structural roles, proteoglycans are important for cell proliferation and differentiation and perform, through binding of growth factors, essential functions in remodeling processes. ${ }^{11}$ Hyaluronan is a major carbohydrate component of the extracellular matrix that can be found in skin, joints, and 
Wharton jelly in the umbilical cord. ${ }^{12}$ Hyaluronan is known to provide mechanical support and to interact with different growth factors. ${ }^{13}$

In the human cervix alterations in the proteoglycans are known to play an important role in the ripening of the cervix that takes place during the last weeks of pregnancy and during labor. ${ }^{14,} 15$ Only one study has described the presence of intact proteoglycans in the fetal membranes, and intact proteoglycans in human fetal membranes have never been studied before from an obstetric point of view, so the impact on the rupture of fetal membranes remains unknown. ${ }^{16}$

Until now no obvious mechanism for the change in the biomechanical properties of fetal membranes at term has been found. Our hypothesis is that proteoglycans are involved in this process. To test this hypothesis we studied the contents and distributions of proteoglycans, hyaluronan, and collagen in human fetal membranes to characterize various important structural components of the extracellular matrix in the membranes at term before labor.

\section{Material and methods}

Patient details. Informed consent was obtained from all patients. The ethics committee of Aarhus County, Denmark, approved the study. Fetal membranes were obtained from 10 women undergoing elective cesarean delivery in gestational week 38 (range, 37 weeks 4 days' to 38 weeks 5 days' gestation) for breech presentation, previous rupture of the anal sphincter, history of intrauterine death, or elective repeat cesarean birth. The fetal membranes were collected at delivery, and biopsy specimens were frozen at $-80^{\circ} \mathrm{C}$ immediately after sampling.

Reagents. Alcian blue 8GX was obtained from Wieslab (Wieslab AB, Lund, Sweden). Ultra-pure deoxyribonucleic acid grade agarose came from Bio-Rad Laboratories (Hercules, Calif). Chondroitin ABC (EC 4.2.2.4) lyase was purchased from Seikagaku (Tokyo, Japan). Guanidinium chloride, ovomucoid, Streptomyces hyaluronidase, and pepsin were products of Sigma (St Louis, Mo). Diethylaminoethyl cellulose 52 was a product of Whatman International (Maidstone, United Kingdom). Sodium dodecyl sulfate gels came from Invitrogen (Carlsbad, Calif). The transfer membrane and polyvinylidene difluoride (PVDF) filter came from Millipore Corporation (Watford, United Kingdom). The Vectastain-Elite avidinbiotin complex reagent was purchased from Vector Laboratories (Burlingame, Calif). Chloramine T, ethylenediaminetetraacetic acid (EDTA), and $N$-ethylmalemide were purchased from BDH Laboratory Supplies (Poole, United Kingdom). The Pharmacia HA test was manufactured by Pharmacia Diagnostics (Uppsala, Sweden). All other chemicals were of analytic grade and purchased from local chemical agencies.

Small proteoglycans for standards were purified from cow sclera. ${ }^{17}$ Rabbit polyclonal antibodies against human decorin (LF 51) and biglycan (LF 150) were gifts from Dr Larry Fisher (National Institute of Dental and Craniofacial Research, Bethesda, Md). The polyclonal bovine antibody versican (AK 6) was a gift from Professor Dick Heinegård (University of Lund, Sweden). The monoclonal mouse perlecan antibody was supplied by Zymed Laboratories (San Francisco, Calif).

Preparation of biopsy specimens. Samples of intact amniochorion and separated amniotic and chorionic membranes were prepared from each placenta. Specimens of $1-, 4-$, and $8-\mathrm{cm}^{2}$ areas were punched out from halfway between the placental edge and a point opposite the placenta with a cutting instrument of razor blades in parallel. ${ }^{2}$ Specimens were then rinsed in phosphate-buffered saline solution ( $\mathrm{pH} 7.4$ ) and stored at $-80^{\circ} \mathrm{C}$ to await further analysis.

\section{Analytic procedures}

Collagen determination. Specimens of $1 \mathrm{~cm}^{2}$ were analyzed for hydroxyproline content according to the Stegeman and Stalder method, as described previously elsewhere. ${ }^{18}$

Proteoglycan determination. The specimens were cut into pieces and homogenized with a manual mortar. The samples were extracted in 4-mol/L guanidinium chloride and $0.05-\mathrm{mol} / \mathrm{L}$ acetate $(\mathrm{pH} 5.8)$ supplemented with 1\% Triton-X 100 (Union Carbide Corporation, Danbury, Conn), 0.005-mol/L N-ethylmalemide, 0.01$\mathrm{mol} / \mathrm{L} \varepsilon$-aminocaproic acid, 0.005 -mol/L benzamide hydrochloride, and $0.01-\mathrm{mol} / \mathrm{L}$ EDTA $(50 \mu \mathrm{L}$ extraction buffer per milligram of wet weight of tissue) for 48 hours. After centrifugation at $12,000 \mathrm{~g}$ for 30 minutes at $4^{\circ} \mathrm{C}$ the supernatant was removed and kept at $-80^{\circ} \mathrm{C}$ to await further analysis. The proteoglycans were precipitated with the positively charged alcian blue dye, as described by Björnsson in 1993. ${ }^{19}$, 20 They were then purified, and the total amount of proteoglycans was determined by estimating the absorbency of alcian blue at 600 nm. ${ }^{19}, 20$ The results were expressed in micrograms of proteoglycans per milligram of wet weight of tissue, with small proteoglycans from sclera used as a standard. ${ }^{17}$

Electrophoretic separation. Samples were dissolved in the Laemmli buffer system with $0.5 \%$ (wt/wt) of sodium dodecyl sulfate overnight. Digestion with chondroitinase ABC lyase $(5 \mathrm{U} / \mathrm{mL})$ was carried out as described by Lindblom et $\mathrm{al}^{21}$ in 1989 . The proteoglycans were separated in $2 \%$ agarose gels or with sodium dodecyl sulfate-polyacrylamide gel electrophoresis $3 \%$ to $12 \%$ gradient gels. After electrophoresis the gels were stained with either Coomassie blue or alcian blue and subjected to scanning with a transmission scanner (Acerscan TPO; Acer Inc, Taipei Hsien, Taiwan). The data were analyzed with Phoretix 1D (Nonlinear Dynamics Limited, Newcastle upon Tyne, United Kingdom) software.

Western blots. The proteoglycans were electrophoresed on 2\% (wt/vol) agarose gels, blotted onto a PVDF-P 
Table I. Hydroxyproline in fetal membranes from elective cesarean deliveries at term

\begin{tabular}{|c|c|c|c|c|c|}
\hline & \multirow[b]{2}{*}{ No. } & \multirow[b]{2}{*}{ Extractability* $(\%)$} & \multicolumn{3}{|c|}{ Hydroxyproline } \\
\hline & & & $\mu g / m g d r y$ weight & $\mu \mathrm{g} / \mathrm{mg}$ wet weight & $\mu \mathrm{g} / \mathrm{cm}^{2}$ \\
\hline Intact amniochorion membrane & 10 & $9.7 \pm 1$ & $21 \pm 2$ & $2.8 \pm 0.4$ & $112 \pm 15$ \\
\hline Amnion & 9 & $3.0 \pm 1 \dagger$ & $50 \pm 3 † \neq$ & $6.7 \pm 0.8 \dagger$ & $32 \pm 2 \dagger$ \\
\hline Chorion & 9 & $10 \pm 1$ & $16 \pm 1$ & $2.0 \pm 0.2$ & $60 \pm 6$ \\
\hline
\end{tabular}

Hydroxyproline constitutes approximately $12.4 \%$ of the collagen molecule. Values are given as mean \pm SEM.

$*$ Extractability of collagen in acetic acid with pepsin.

$\dagger P<.001$ (amnion vs chorion).

‡The water contents in both the amnion and the chorion were around $86 \%$.

membrane, and digested with chondroitinase $\mathrm{ABC}$ lyase before immunostaining with antibodies to versican (diluted 1:500), biglycan (1:400), decorin (1:400), or perlecan (1:500). The proteoglycans were finally visualized by means of a secondary peroxidase-conjugated swine antirabbit antibody according to the manufacturer's instructions (DAKO A/S, Glostrup, Denmark).

Hyaluronan determination. An aliquot of the guanidinium extract was diluted 20 times in buffered urea (6-mol/L urea and 0.05-mol/L acetic acid, pH 5.8; complemented with $0.01-\mathrm{mol} / \mathrm{L}$ EDTA, $0.005-\mathrm{mol} / 1 \mathrm{~N}-$ ethylmalemide, and $5 \mu \mathrm{g} / \mathrm{mL}$ ovalbumin). The fraction containing hyaluronan was eluted with $0.6-\mathrm{mol} / \mathrm{L}$ acetic acetate in urea, pH 5.8 (complemented with $0.005-\mathrm{mol} / \mathrm{L}$ $N$-ethylmalemide, $0.01-\mathrm{mol} / \mathrm{L}$ EDTA, and $5 \mu \mathrm{g} / \mathrm{mL}$ ovalbumin), on columns of diethylaminoethyl $52(0.5 \times 0.7$ $\mathrm{cm})$. Hyaluronan was then quantitated by means of a radioimmunoassay. ${ }^{22}$

Interassay and intra-assay variations and sample variation. In evaluating the alcian blue technique combined with gel scanning we measured the total proteoglycan content and found an intra-assay coefficient of variation of $3.6 \%$ ( $\mathrm{n}=$ $6)$. The interassay coefficient of variation was $7 \%(n=6)$.

We measured the sample variation and biologic variation within the same membrane by taking 3 samples from each membrane, all within the same distance from the placental edge $(n=5)$. This showed a variation of $15 \%$ (see Fig 2). Only 1 specimen was then used in the following experiments.

Immunohistochemical studies (decorin and biglycan). Fetal membrane strips were rolled with the amnion innermost, placed in $3 \%$ glutaraldehyde with $5 \%$ sucrose added, and fixed for 24 hours before processing and mounting in paraffin wax. Sections of $3 \mu \mathrm{m}$ were digested with chondroitinase $\mathrm{ABC}$ overnight, incubated with swine serum (diluted 1:20) for 20 minutes, and incubated with primary antibody (diluted 1:200 in tris[hydroxymethyl] aminomethane [Tris] hydrochloride, $\mathrm{pH}$ 7.6 , with $2 \%$ bovine serum albumin) for 30 minutes. The secondary antibody, swine antirabbit, was then added for 30 minutes, and reactivity was detected with avidin-biotin vectorstatin AK-500 followed by vector red (vector SK 5100). The background was stained with
Mayer hematoxylin. Negative control slides were incubated with bovine serum albumin and no primary antibody.

Histochemical studies (hyaluronan). The fixation was carried out as described previously. The isolation and biotin labeling of the hyaluronan-binding protein have been described in detail elsewhere. ${ }^{23}$ The sections were incubated in 3\% hydrogen peroxide in methanol for 5 minutes and blocked with $1 \%$ bovine serum albumin for 30 minutes. The sections were then incubated with $100 \mu \mathrm{L}$ of the biotinylated hyaluronan-binding protein (1:40) overnight, followed by incubation with the VectastainElite avidin-biotin complex reagent (1:200) for $40 \mathrm{~min}$ utes. Finally, the sections were incubated in $0.1 \%$ diaminobenzidene and $10 \mu \mathrm{L}$ hydrogen peroxide (from $30 \%$ stock) in $10 \mathrm{~mL}$ of $0.05-\mathrm{mol} / \mathrm{L}$ Tris hydrochloride buffer, pH 7.6. The background was stained with Mayer hematoxylin. Negative control slides were incubated with $50 \mathrm{U} / \mathrm{mL}$ Streptomyces hyaluronidase.

Statistical analysis. All results were expressed as mean \pm SEM. The data values were normally distributed, and comparisons were made between amnion and chorion with the Student $t$ test. The level of significance used was $P \leq .05$.

\section{Results}

Collagen. Fetal membranes were found to be collagenrich tissues, and the amnion in particular contained an especially high concentration of collagen as calculated from the hydroxyproline concentration (Table I). Per weight unit the chorion contained only a third as much collagen as was found in the amnion. Moreover, the nonextractable part of collagen in the amnion was significantly higher than that in the chorion. Per square unit the amnion contained only half the amount of collagen found in the chorion. Because the amnion is $<25 \%$ the thickness of the chorion, ${ }^{4}$ however, the amount of collagen per square unit in the amnion indicated an extremely high level.

Proteoglycans. The proteoglycans were precipitated and separated with agarose gel electrophoresis. The amniochorial membrane was clearly dominated by smaller proteoglycans (Figs 1 and 2). Digestion with ABC chon- 


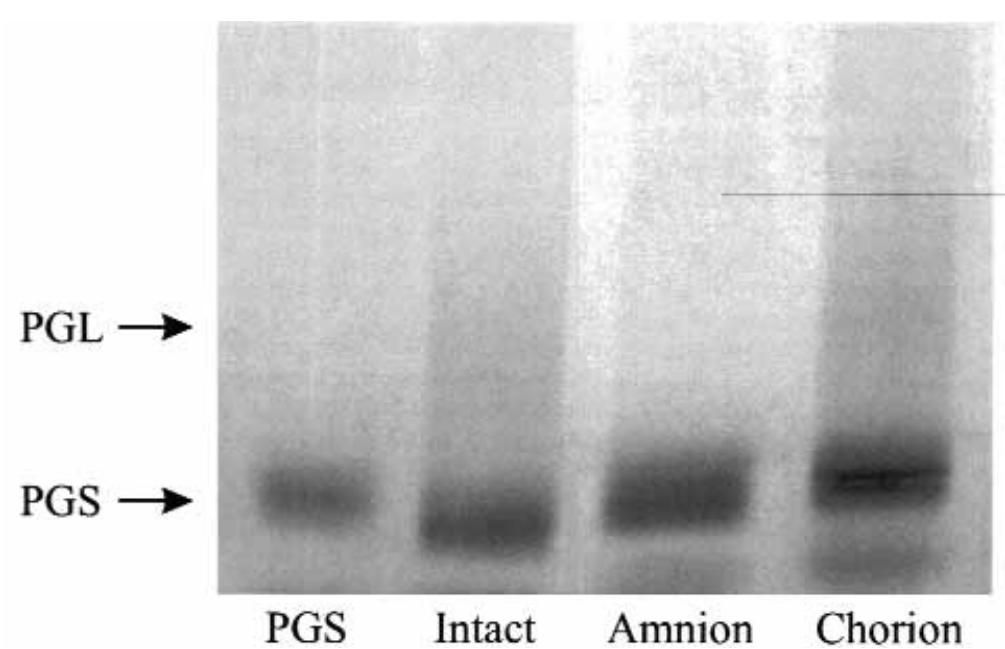

Fig 1. Analysis of proteoglycans from 1 fetal membrane on $2 \%$ agarose gel. Lane 1, Small proteoglycans (PGS) from bovine sclera used as standard; lane 2, intact amniochorion membrane; lane 3, amnion; lane 4, chorion. Upper band, Large proteoglycans (PGL); lower band, smaller proteoglycans (PGS).

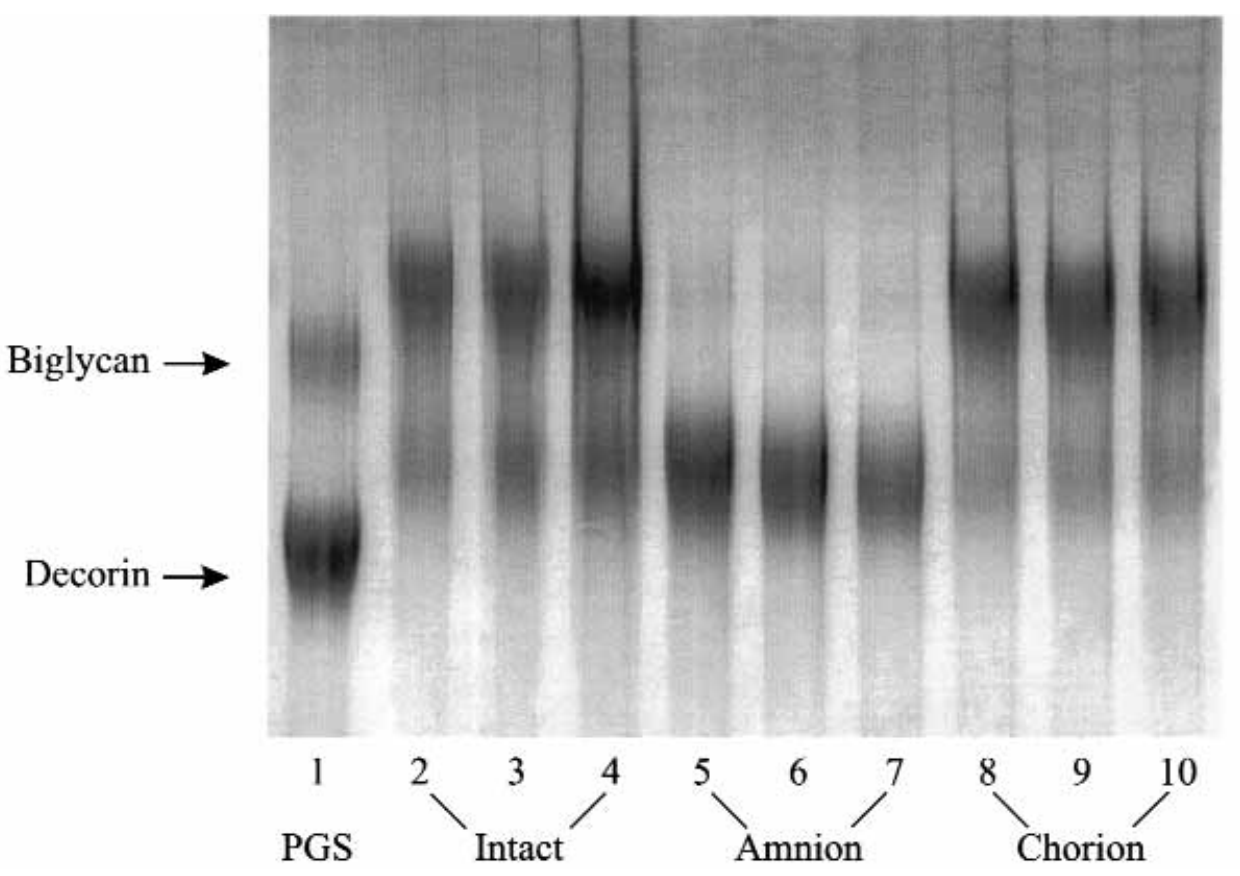

Fig 2. Analysis of small proteoglycans decorin and biglycan in 1 fetal membrane on sodium dodecyl sulfatepolyacrylamide gel electrophoresis gradient gel (4\%-12\%). Lane 1, Small proteoglycans (PGS) from bovine sclera used as standard; lanes 2 to 4, intact amniochorion membrane from 3 different locations; lanes 5 to 7 , amnion from 3 different locations; lanes 8 to 10, chorion from 3 different locations. All tissue samples were collected within same distance from placental edge. Alcian blue was used for analyzing amounts of proteoglycan. Impact of biglycan may be overestimated, because 2 side chains of biglycan may bind twice as much alcian blue as can decorin.

droitinase lyase removed $>95 \%$ of the material (data not shown). The smaller proteoglycans in the amnion and chorion were identified as decorin and biglycan by Western blotting. The large proteoglycans versican and perlecan were detected only in very small amounts in the chorion and were not studied in more detail (data not shown).
We estimated the concentration of biglycan and decorin by combining the total amount of proteoglycans per milligram wet weight determined by alcian blue with gel scanning. The total amounts of proteoglycans in the amnion and chorion per milligram of wet weight were similar. The amnion was dominated by decorin, which constituted two thirds of the total amount of proteogly- 

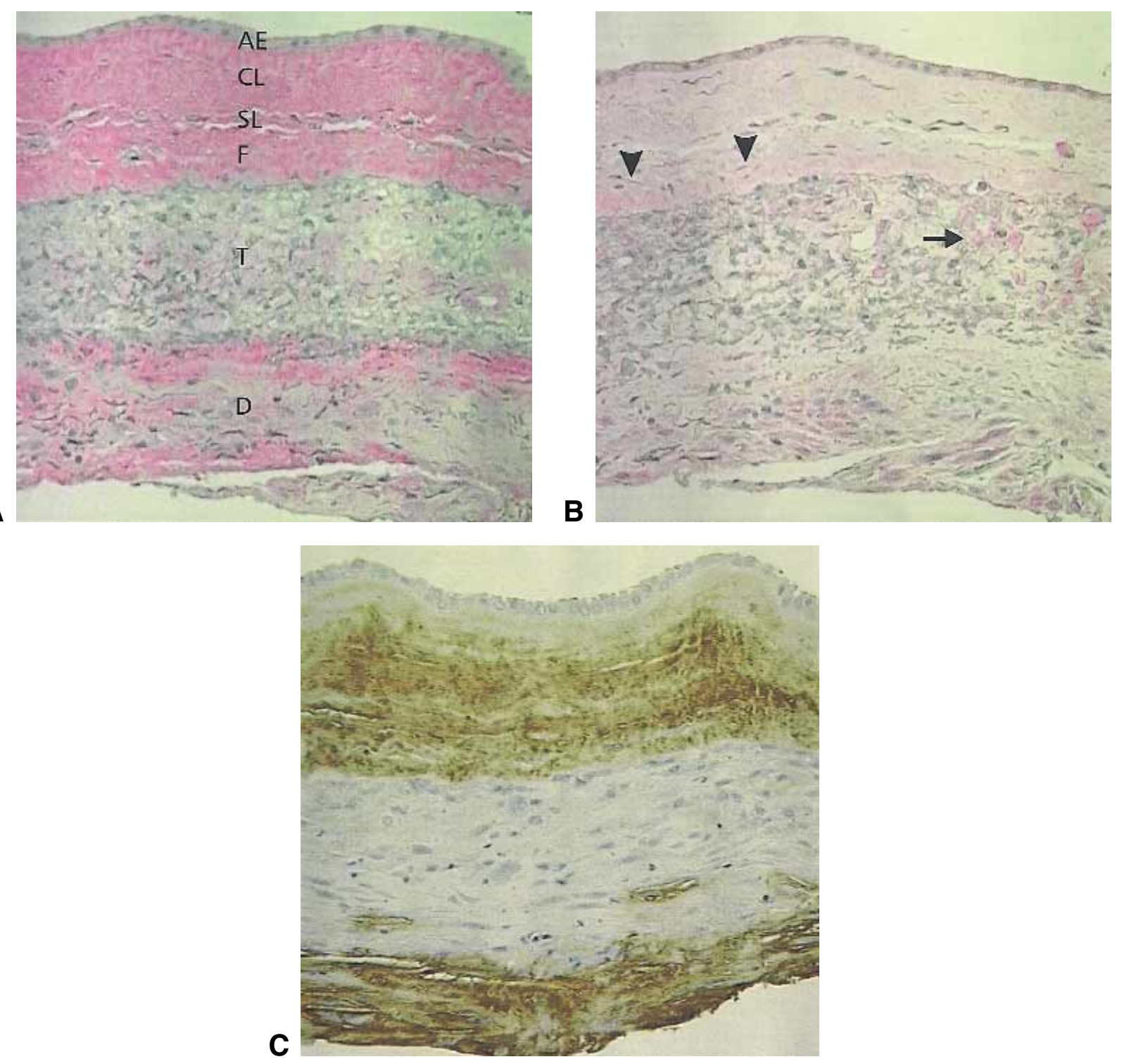

Fig 3. A, Immunostaining for decorin, also demonstrating layers of amniochorion membrane. Single layer of amniotic epithelium $(A E)$ rests on amniotic basement membrane. Compact layer of amnion $(C L)$ is densely packed. Spongy layer $(S L)$ is interface between amnion and chorion. Chorion consists of fibroblastic $(F)$ and trophoblastic $(T)$ parts. Decidual cell layers $(D)$ underlie chorion. B, Immunostaining for biglycan shows distinct staining in trophoblast layer (arrow) and amniotic epithelium and diffuse staining of fibroblastic part of chorion (arrowheads). C, Histochemical staining for hyaluronan with biotin-labeled hyaluronan-binding protein. (A-C: Original magnification $\times 10$.)

cans (Table II), with the rest consisting of biglycan (Table II and Fig 2, lanes 5-7). This was also well illustrated by immunohistochemical studies, in which decorin was localized in close connection with the collagen fibers in the amnion (Fig 3, A), whereas biglycan appeared predominantly in and close to the amniotic epithelium (Fig 3, B). In quantitative terms the chorion contained mostly biglycan (Table II and Fig 2, lanes 810). Immunohistochemical studies showed that the chorion was composed of a fibroblastic part rich in decorin and a trophoblastic part rich in biglycan. The fibroblastic part of the chorion displayed sparse staining for biglycan (Fig 3, B). The decidual tissue stained positively for both decorin and biglycan.

Hyaluronan. A high concentration of hyaluronan was detected in the amnion, and smaller amounts were found in the chorion (Table II). The appearance of hyaluronan was closely connected with the fibrous parts of both the amnion and the chorion, where the staining was considerable (Fig 3, C). The most intense staining occurred at the interface between the amnion and the chorion, known as the spongy layer. The amniotic epithelium and the trophoblasts did not stain for hyaluronan at all, whereas the decidual cell layers revealed considerable staining. 
Table II. Decorin, biglycan, and hyaluronan per milligram of wet weight in fetal membranes from elective cesarean deliveries at term $(\mathrm{N}=10)$

\begin{tabular}{lccc}
\hline & Decorin $(\mu g / m g$ wet weight $)$ & Biglycan $(\mu g / m g$ wet weight $)$ & Hyaluronan $(\mu g / m g$ wet weight $)$ \\
\hline Intact amniochorion membrane & $1.0 \pm 0.1$ & $1.9 \pm 0.4$ & $1.1 \pm 0.2$ \\
Amnion & $1.8 \pm 0.3^{*}$ & $0.9 \pm 0.3 \dagger$ & $2.9 \pm 0.6 \dagger$ \\
Chorion & $0.9 \pm 0.1$ & $2.0 \pm 0.3$ & $1.1 \pm 0.2$ \\
\hline
\end{tabular}

Values are given as mean \pm SEM

$* P<.001$, versus chorion.

$\dagger P<.05$, versus chorion.

\section{Comment}

In this study we have found the fetal membranes to consist of two differently composed tissues, the fibrous decorin-rich amnion and the less homogeneous fibrous, cellular, and biglycan-rich chorion. The appearance of decorin in close connection with the collagen fibers supports the hypothesis of an interaction between decorin and collagen, as reported by others. ${ }^{7,} 24$ This interaction is probably of importance for the functional properties of the collagen fibrils in the amnion and the fibrillar part of the chorion. The presence of large amounts of biglycan in the chorion is of considerable interest. Because biglycan has a much weaker interaction with collagen $\mathrm{I}$, it should result in a different extracellular matrix, as is clear in Fig 3, $A$ and $B$. Other functional differences generated by this alternative organization must await further investigation.

The concentration and distribution of collagen found in this study correspond well to what others have reported for term fetal membranes concerning the high amount of collagen in the amnion. ${ }^{4-6}$ In contrast to this finding, Schwarzfurtner et $\mathrm{al}^{25}$ found the same amount of collagen in the amnion as in the chorion; however, the cellular parts of the chorion had been removed before analysis. The lower extractability by pepsin digestion of the collagen in the amnion, demonstrating many crosslinks, indicates that the function of this collagen is to resist tensile forces. The high content of decorin further supports this function and also forms the basis for a rapid remodeling process toward the time of labor.

Hyaluronan was localized in abundant amounts in the fibrous parts of both the amnion and the chorion. Others have found a similarly high concentration of hyaluronan in the amnion and have also described the fact that the concentration of hyaluronan increases with gestational age. ${ }^{26}$ It has been proposed that the induction of a gelatinous substance in the spongy layer between the amnion and the chorion at term may result in dramatic changes in the mechanical properties of the tissue. ${ }^{2}$ In accordance with this hypothesis, our study showed intense staining for hyaluronan at the interface between the amnion and chorion (Fig 3, C).

The mechanism involved in membrane rupture could be a weakening of the interaction between collagen and decorin. Hyaluronan possesses the ability to create a great swelling pressure of the tissue because of its high water-binding capacity. A combination of these processes could separate the amnion and the chorion, thereby explaining the dramatic decrease in the mechanical strength, because the amnion and the chorion would no longer be able to work in parallel. Furthermore, hyaluronan may be present in the intermediate layer between the amnion and chorion, where it would act as a gliding layer during the birth process and thereby protect the fetus.

Characterization of various important extracellular matrix components in these tissues gives us the opportunity to study the impact of gestational age and parturition on these matrix molecules. It will be of great interest to study the impact of a possible change in composition of the proteoglycans on the tensile strength of the membranes. It will also be possible to study whether local changes are taking place in the part of the fetal membranes overlaying the cervix and in cases of preterm prelabor rupture of membranes. In future studies it may be interesting to test the hypothesis that a maturation process similar to the inflammatory ripening process occurring in the cervix is going on in the fetal membranes. ${ }^{14}$

We thank Tove Stenum, Karin Barchan, Iris Gusstavsson, and Cathrine Johannsson for their excellent technical support.

\section{REFERENCES}

1. Gibbs RS RR, Hiller SL, Eschenbach DA, Sweet RL. A review of premature birth and subclinical infection. Am J Obstet Gynecol 1992;166:1515-28.

2. Helmig R, Oxlund H, Petersen LK, Uldbjerg N. Different biomechanical properties of human fetal membranes obtained before and after delivery. Eur J Obstet Gynecol Reprod Biol 1993;48:183-9.

3. Malak TM, Ockleford CD, Bell SC, Dalgleish R, Bright N, Macvicar J. Confocal immunofluorescence localization of collagen types I, III, IV, V and VI and their ultrastructural organization in term human fetal membranes. Placenta 1993;14:385-406.

4. Halaburt JT, Uldbjerg N, Helmig R, Ohlsson K. The concentration of collagen and the collagenolytic activity in the amnion and the chorion. Eur J Obstet Gynecol Reprod Biol 1989;31: $75-82$.

5. Evaldson GR, Larsson B, Jiborn H. Is the collagen content reduced when the fetal membranes rupture? A clinical study of term and prematurely ruptured membranes. Gynecol Obstet Invest 1987;24:92-4. 
6. Skinner SJ, Campos GA, Liggins GC. Collagen content of human amniotic membranes: effect of gestation length and premature rupture. Obstet Gynecol 1981;57:487-9.

7. Uldbjerg N, Danielsen CC. A study of the interaction in vitro between type I collagen and a small dermatan sulphate proteogly can. Biochem J 1988;251:643-8.

8. Danielson KG, Baribault H, Holmes DF, Graham H, Kadler KE, Iozzo RV. Targeted disruption of decorin leads to abnormal collagen fibril morphology and skin fragility. J Cell Biol 1997;136: 729-43.

9. Roughley PJ, Lee ER. Cartilage proteoglycans: structure and potential functions. Microsc Res Tech 1994;28:385-97.

10. Ruoslahti E. Structure and biology of proteoglycans. Annu Rey Cell Biol 1988;4:229-55.

11. Iozzo RV, Murdoch AD. Proteoglycans of the extracellular environment: clues from the gene and protein side offer novel perspectives in molecular diversity and function. FASEB J 1996; 10:598-614.

12. Sobolewski K, Bańkowski E, Chyczewski L, Jaworski S. Collagen and glycosaminoglycans of Wharton's jelly. Biol Neonate 1997 71:11-21.

13. Westergren-Thorsson G, Särnstrand B, Fransson LA, Malmström A. TGF-beta enhances the production of hyaluronan in human lung but not in skin fibroblasts. Exp Cell Res 1990;186:192-5.

14. Winkler M, Rath W. Changes in the cervical extracellular matrix during pregnancy and parturition. J Perinat Med 1999;27:45-60

15. Westergren-Thorsson G, Norman M, Björnsson S, Endrésen U, Stjernholm Y, Ekman G, et al. Differential expressions of mRNA for proteoglycans, collagens and transforming growth factorbeta in the human cervix during pregnancy and involution. Biochim Biophys Acta 1998;1406:203-13.

16. Brennan MJ, Oldberg A, Pierschbacher MD, Ruoslahti E. Chondroitin/dermatan sulfate proteoglycan in human fetal mem- branes. Demonstration of an antigenically similar proteoglycan in fibroblasts. J Biol Chem 1984;259:13742-50.

17. Cöster L, Fransson LA. Isolation and characterization of dermatan sulphate proteoglycans from bovine sclera. Biochem J 1981;193:143-53.

18. Uldbjerg N, Ekman G, Malmström A, Ulmsten U, Wingerup L Biochemical changes in human cervical connective tissue after local application of prostaglandin E2. Gynecol Obstet Invest $1983 ; 15: 291-9$

19. Björnsson S. Size-dependent separation of proteoglycans by electrophoresis in gels of pure agarose. Anal Biochem 1993;210: 292-8.

20. Björnsson S. Simultaneous preparation and quantitation of proteoglycans by precipitation with alcian blue. Anal Biochem 1993;210:282-91.

21. Lindblom A, Carlstedt I, Fransson LA. Identification of the core proteins in proteoglycans synthesized by vascular endothelial cells. Biochem J 1989;261:145-53.

22. Brandt R, Hedlöf E, Asman I, Bucht A, Tengblad A. A convenient radiometric assay for hyaluronan. Acta Otolaryngol Suppl Stockh 1987;442:31-5.

23. Hellström S, Tengblad A, Johansson C, Hedlund U, Axelsson E. An improved technique for hyaluronan histochemistry using microwave irradiation. Histochem J 1990;22:677-82.

24. Hedbom E, Heinegård D. Interaction of a 59-kDa connective tissue matrix protein with collagen I and collagen II. J Biol Chem 1989;264:6898-905.

25. Schwarzfurtner VH, Huttegger I, Schmidt W, Sattler K. Quantitative Untersuchungen über den Gehalt an Kollagen im menschllichen Amnion und Chorion. Anat Anz 1986;161:3-9

26. Skinner SJ, Liggins GC. Glycosaminoglycans and collagen in human amnion from pregnancies with and without premature rupture of the membranes. J Dev Physiol 1981;3:111-21. 